\title{
Strength and rigidity of the masonry fragment in a fuzzy setting
}

\author{
Vladimir Adishchev ${ }^{1, *}$, Irina Kucherenko ${ }^{1,2}$, and Maria Teterina ${ }^{1}$ \\ ${ }^{1}$ NSUACE (Sibstrin), 630008, 113, Leningradskaya st., Novosibirsk, Russia \\ ${ }^{2}$ NHMCS 630117, 49, Ivanoya st., Novosibirsk, Russia
}

\begin{abstract}
The strength and stiffness of masonry depend on various factors, such as the properties of the brick and mortar, their specific volume, relative position and quality of adhesive bonds. A high degree of uncertainty of these factors and a large scatter of experimental data in the testing of bricks and mortar are the important features of the masonry. In this paper, we present a mathematical model that allows us to determine the averaged characteristics of masonry based on the mechanical properties of substructural materials and their specific volume content in a fuzzy setting. All input parameters allowing to obtain fuzzy estimates of stresses and deformations of a brickwork fragment are determined by membership functions. For a representative volume of masonry, dependencies are obtained that allow to determine the level of destructive loads and the nature of destruction.
\end{abstract}

\section{Introduction}

According to the latest design rules [1], brick masonry is considered to be a uniform material with piecewise constant elasticity modulus, which depends on its strength characteristics. The series of runs demonstrate [2-4] that brickwork with various types of loading exhibits a pronounced anisotropy, its deformative and strength properties depend on a number of factors such as the physico-mechanical properties of the components, their specific volume content, the quality of adhesive bonds. Therefore, there is a need to develop an adequate mathematical model that allows obtaining the mechanical characteristics of a fragment of masonry in various directions or their interval estimates. Beside, there is a necessity for taking into account the properties of substructural materials.

The materials forming the brickwork are distinguished by a high degree of uncertainty of their mechanical properties. For example, in [2], a sufficiently large scatter in strength and stiffness was experimentally shown for specimens of tuff $(50 \mathrm{~cm} \times 60 \mathrm{~cm} \times 20 \mathrm{~cm})$ tested for compression. It is emphasized that the dispersion of results increases significantly within changing over model to real structure. Thus, it is necessary to take into account the uncertainty of the input data to assess the strength and rigidity of the brickwork.

Classical approach of the probability calculus requires large computational resources. The complete solution of practical problems in a probabilistic formulation involves the

\footnotetext{
*Corresponding author: adishch@mail.ru
} 
representation of random variables that form an initial data packet by defining probability distribution functions, their numerical characteristics (expectation, variance, etc.).The combination of known distribution functions is rather limited. In addition, obtaining solutions to problems in the form of a combination of random variables is an extremely complex mathematical task. In our opinion, the simplest and most convenient in solving practical problems is the application of the fuzzy set theory and fuzzy logic $[5,6]$. When solving problems in fuzzy formulation, the initial data are represented in the form of membership functions, which are analogous to the density of probability distribution, and it does not require its analytical representation [7]. The algebra of fuzzy numbers makes it easy to operate with fuzzy values.

The proposed approach to the construction of a mathematical model involves the description of a brick mechanical characteristics and mortar as fuzzy values. Their specific volumetric content and quality of adhesive bonds, strength and stiffness are effectively described by the membership functions obtained on the basis of experimental data processing.

\section{Structural model}

To determine the effective elastic characteristics of the brickwork, the mathematical model described in [8] was used. It is assumed the material has a periodic structure, the interfaces between the substructural components are parallel to some Cartesian coordinate system, and there is no separation and slippage. The material is modeled by the characteristic continuously repeating element shown in Fig. 1.

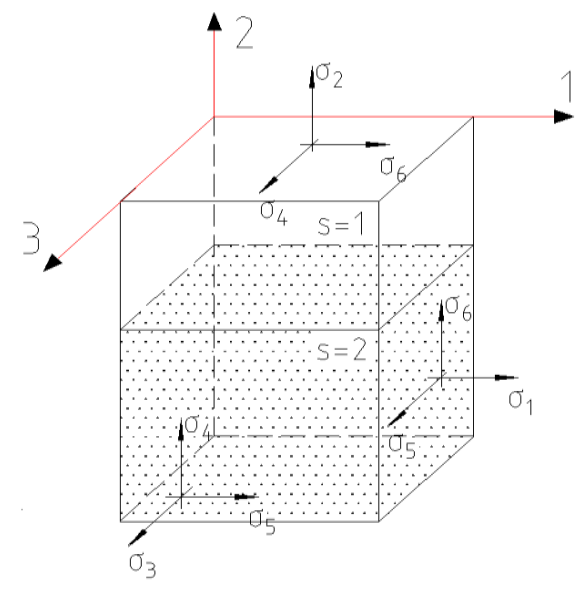

Fig. 1. The characteristic structural element of a nonhomogeneous material.

The material of each component $\mathrm{s}$ is considered orthotropic and obeys the generalized Hooke's law [8]:

$$
\varepsilon^{(s)}=A^{(s)} \sigma^{(s)} \quad(s=1,2),
$$

where $A^{(s)}$ - flexibility matrix for each component $s$ of the medium. 


$$
A^{(s)}=\left[\begin{array}{cccccc}
a_{11}^{(s)} & a_{12}^{(s)} & a_{13}^{(s)} & & 0 & \\
a_{21}^{(s)} & a_{22}^{(s)} & a_{23}^{(s)} & & 0 & \\
a_{31}^{(s)} & a_{32}^{(s)} & a_{33}^{(s)} & & 0 & \\
& & & a_{44}^{(s)} & 0 & 0 \\
0 & 0 & 0 & 0 & a_{55}^{(s)} & 0 \\
& & & 0 & 0 & a_{66}^{(s)}
\end{array}\right], \quad \begin{gathered}
a_{i j}^{(s)}=\frac{-v_{j i}^{(s)}}{E_{j}^{(s)}},(i, j=1,2,3) \\
a^{(s)}=\frac{1}{E_{l}^{(s)}},(l=4,5,6)
\end{gathered}
$$

$\sigma^{(s)}$ is the generalized stress matrix, $\varepsilon^{(s)}$ is the generalized deformation matrix for each component $s$ of the nonhomogeneous material.

Correctly formulated conjugation conditions on the partition planes between the substructural components are used to construct the constitutive relations of the composite by the method described in [8]. The generalized Hooke's law for the characteristic element (Fig. 1) is obtained in analytical form:

$$
\sigma_{j}=\sum_{k=1}^{3} B_{j k} \varepsilon_{k}, \sigma_{l}=B_{l l} \varepsilon_{l}, \quad(j=1,2,3 ; \quad l=4,5,6)
$$

where $\sigma_{j}, \sigma_{l}$ are elements of the stress matrix, $\varepsilon_{k}, \varepsilon_{l}-$ are the elements of the deformations matrix of a structurally inhomogeneous material, $B$ - generalized stiffness matrix of a structurally inhomogeneous material whose elements are obtained in [8]. For instance, $B_{11}=\frac{\left(C_{12}\right)^{2}}{C}+\sum_{s=1}^{2} \frac{\omega_{s} a_{33}^{(s)}}{\left|A_{22}^{(s)}\right|}, C=\sum_{s=1}^{2} \frac{\omega_{s}\left|A^{(s)}\right|}{\left|A_{22}^{(s)}\right|}, C_{12}=\sum_{s=1}^{2} \frac{\omega_{s}\left|A_{23}^{(s)}\right|}{\left|A_{22}^{(s)}\right|}$.

To assess the strength of each component $s$ from equations (1) - (3) and the accepted conjugation conditions at the interfaces, we can get expressions for stresses:

$$
\begin{gathered}
\sigma_{1}^{(s)}=\frac{1}{\left|A_{22}^{(s)}\right|}\left(a_{33}^{(s)} \varepsilon_{1}-a_{13}^{(s)} \varepsilon_{3}+\left|\mathrm{A}_{12}^{(s)}\right| \sigma_{2}\right) \\
\sigma_{2}^{(s)}=\sigma_{2} \\
\sigma_{3}^{(s)}=\frac{1}{\left|\mathrm{~A}_{22}^{(s)}\right|}\left(a_{11}^{(s)} \varepsilon_{3}-a_{13}^{(s)} \varepsilon_{1}+\left|\mathrm{A}_{23}^{(s)}\right| \sigma_{2}\right)
\end{gathered}
$$

где $\left|A_{i j}^{(s)}\right|, i, j=1,2,3$ - algebraic complements of the elements $a_{i j}^{(s)}$ matrix material pliability component $s$ from (2).

Thus, formulas (1) - (4) allow us to obtain the generalized Hooke's law for a heterogeneous material from the known mechanical characteristics of the substructural components, and then for each level of the sample loading, determine the magnitude of the stresses in the structural elements.

\section{Consideration of the uncertainty in the mechanical properties of a brickwork}

An important feature of masonry is a high degree of uncertainty in the mechanical properties of its components and the entire structure. According to [9], permissible deviations from the nominal dimensions of the brick lead to a change in its volume by $8.9 \%$. The average tensile strength of a brick has a spread of up to $25 \%$, therefore, the initial modulus of elasticity associated with it according to [1] by the dependence: 


$$
E_{0}=\alpha R_{u},
$$

is also a fuzzy value. $E_{0}$ is the initial modulus of elasticity of masonry, $R_{u}$ is the temporary resistance (average tensile strength) during compression of masonry, $\alpha-$ is the elastic characteristic depending on the type of masonry.

It is known that the deformative properties of a mortar are not regulated by norms, depend on hardening conditions, and have a large scatter of experimental data [10]. The technological features of the construction of brickwork contribute to the increase of fuzzy defined values, such as the thickness of mortar joints, the number of voids in vertical joints, the change in the properties of the mortar in the contact zone with the brick due to water adsorption [11]. In the present paper, it is proposed to consider the mechanical characteristics of materials and their specific volume content as fuzzy quantities. Their membership functions are constructed on the basis of the processing of experimental data and empirical relationships. Triangular membership function is the most simple:

$$
\mu(a, c)=\left\{\begin{array}{l}
0, u \leq a \bigvee u \geq c \\
\frac{u-a}{b-a}, a<u \leq b \\
\frac{c-u}{c-b}, b<u<c
\end{array}\right.
$$

For fuzzy numbers represented in the form of a set of $\alpha$-sections in accordance with the generalized Zadeh principle [7], a fuzzy-number algebra has been developed, and with simple calculations it is possible to obtain membership functions for the quantities (3) - (6). Then the relations (2) are represented as fuzzy relations.

To account the nonlinear properties of the masonry components, the elements of the flexibility matrix (2) for each loading level are converted to:

$$
\begin{gathered}
\bar{a}_{i i}^{(s)}=\frac{1}{\varphi E_{0}(s)}, \\
\bar{a}_{i j}^{(s)}=-\frac{\psi v_{0}{ }^{(s)}}{\varphi E_{0}{ }^{(s)}},(i, j=1,2,3, i \neq j) \\
\left.\bar{a}_{l l}^{(s)}=\frac{\varphi E_{0}(s) \psi v_{0}(s)}{2\left(1+\psi v_{0}(s)\right.}\right)
\end{gathered}
$$

where $\varphi, \psi$ are piecewise linear functions of the influence of the relative value of compressive stresses $\sigma / \mathrm{R}$ on the value of the average elastic modulus and Poisson's ratio obtained on the basis of experimental data [10]:

$$
\varphi=f_{1}\left(\frac{\sigma}{R}\right), \quad \psi=f_{2}\left(\frac{\sigma}{R}\right)
$$

where $R$ is the compression strength characteristics, $\sigma$ is a stress in the material.

In general, the calculation algorithm consists of several steps. Firstly, generalized (effective) characteristics are determined. Secondly, the generalized stress state is determined. Thirdly, the stress state components of the materials are determined. Further, the criteria for the strength of the components are formulated, if necessary, the destroyed components are removed from the structure. At the final step, the structural model is analyzed and, if necessary, adjusted. A similar approach is used in [12] to study the behavior of concrete under load and is called «take-and-place method». 


\section{Calculation of the load-bearing capacity of the masonry fragment}

As an example, we have considered the masonry fragment from a full-bodied brick of M100 grade and a mortar of grade M75 under uniaxial compression. The substructural materials are brick, mortar of the horizontal and vertical joints, mortar is in the contact zone between brick and horizontal joint [13]. The size of the fragment is $1030 \times 1050 \times 250 \mathrm{~mm}$, it is shown in Fig. 2.

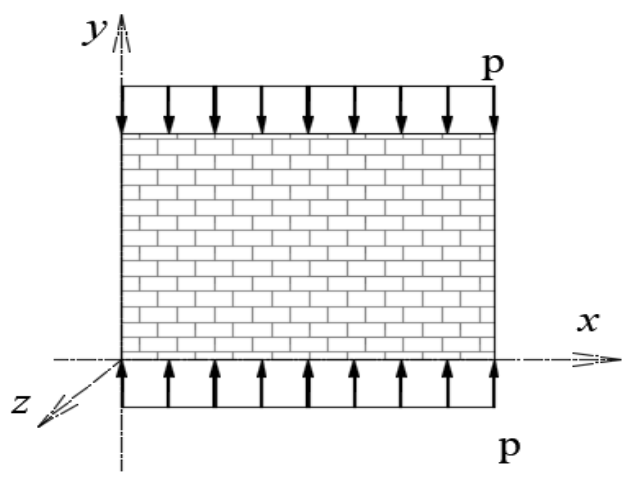

Fig. 2. The masonry fragment.

The calculations were performed using the developed program in C\#. Using expressions (1) - (3), (7), (8), we obtained the strain diagrams shown in Fig. 3. All input parameters are described by triangular membership functions of the form (6). This chart allows to assess the load-bearing capacity and deformations for each level of stresses in the brickwork fragment. Shaded areas are corresponded to possible deformations.

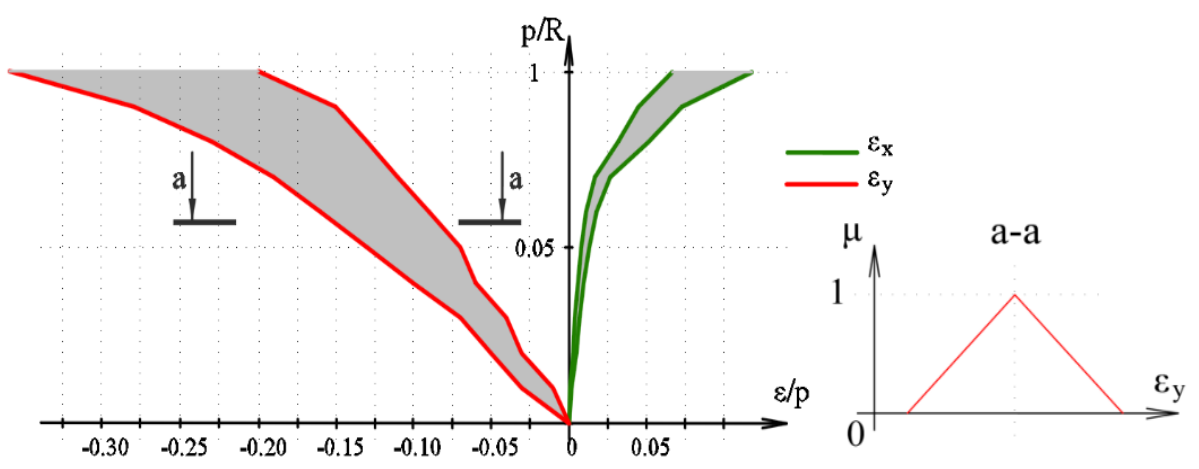

Fig. 3. The stress-strain relationship is under uniaxial compression.

Using the obtained deformation diagram and formulas (4), fuzzy estimates are obtained in substructural materials for compressive stresses in the y-axis direction (Fig. 4) and tensile stresses in the x-axis direction (Fig. 5). The strength limits of masonry, brick and mortar are shown in table 1 according to $[1,9]$. 
Table 1.

\begin{tabular}{|c|c|c|c|c|c|c|}
\hline & \multicolumn{3}{|c|}{$\begin{array}{c}\text { Compression strength } \\
\text { characteristics, MПa }\end{array}$} & \multicolumn{3}{c|}{ Tensile strength, MПа } \\
& $\mathrm{R}_{\min }$ & $\mathrm{R}$ & $\mathrm{R}_{\max }$ & $\mathrm{R}_{\min }$ & $\mathrm{R}$ & $\mathrm{R}_{\max }$ \\
\hline & - & 1,7 & - & 0,16 & 0,18 & 0,2 \\
\hline Masonry & 7,5 & 10 & 12,5 & 1,1 & 2,2 & 3,3 \\
\hline Brick & 6,8 & 8 & 10 & - & 0,16 & - \\
\hline $\begin{array}{c}\text { Vertical joint } \\
\text { mortar }\end{array}$ & 6,8 & 8 & 10 & - & 0,08 & - \\
\hline $\begin{array}{c}\text { Horizontal } \\
\text { joint mortar }\end{array}$ & & & & & & \\
\hline
\end{tabular}

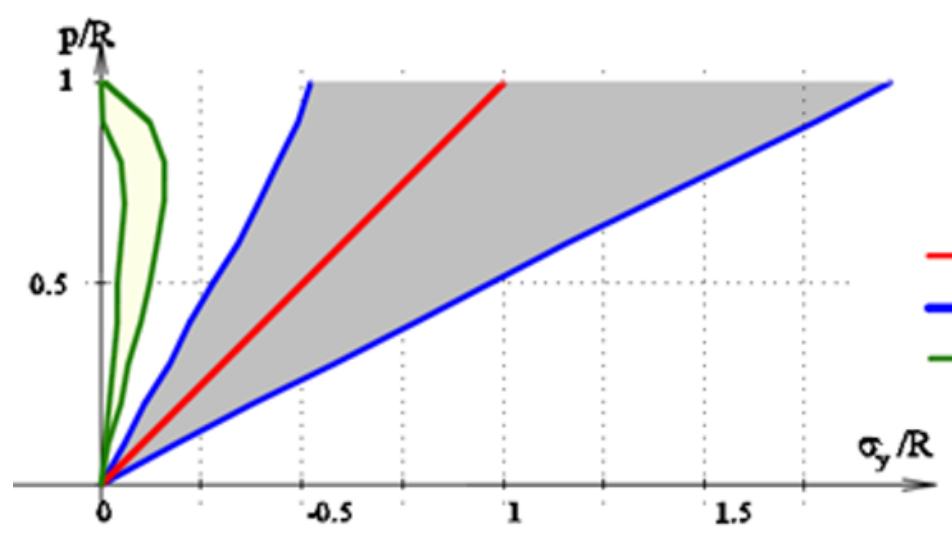

Fig. 4. Compressive stress dispersion in brick and vertical joint mortar.

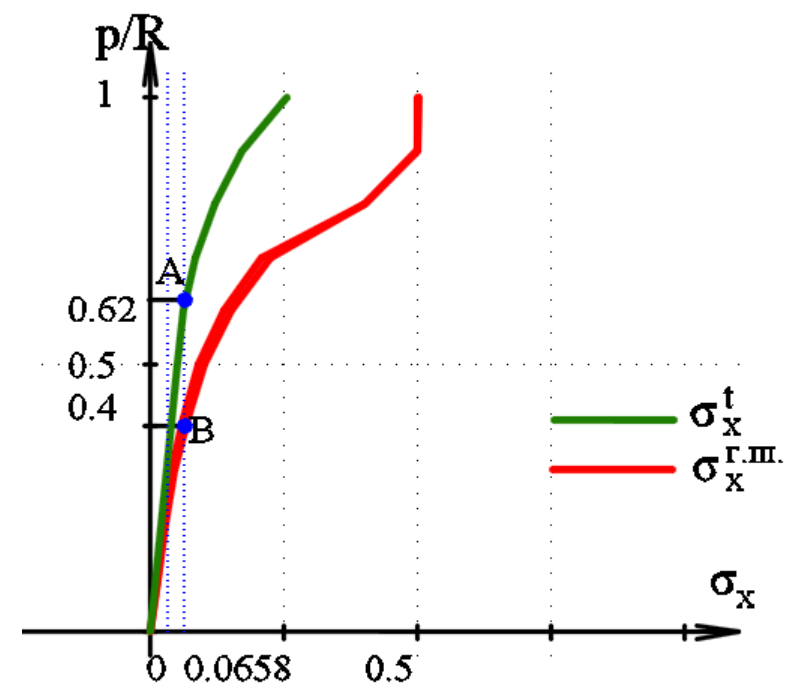

Fig. 5. Tensile stresses in a horizontal row of masonry and horizontal joint mortar.

The obtained fuzzy estimates show that the dispersion of vertical stresses in the brick is quite large, failure begins in a vertical seam (Fig. 4). The dispersion of tensile horizontal stresses is insignificant (Fig. 5), point A corresponds to the tensile strength of the masonry 
on the bandaged section. Point B corresponds to the ultimate strength of the masonry on a non-bandaged section (in a horizontal seam), the stresses of which are $40 \%$ of the breaking stress $\mathrm{R}$, which correspond to the stages of masonry failure in $[14,1]$. The first cracks in the mortar of vertical joints appear at a load of $0.4 \mathrm{R}$. When the load is equal to $0.7 \mathrm{R}$, the cracks are combined, the masonry is destroyed, further operation is impossible.

\section{Conclusion}

The conducted researches allow to represent the following conclusions.

The materials forming the masonry have a high degree of uncertainty in the mechanical properties, which must be taken into account. In addition, it is necessary to introduce not one stochastic quantity, but a whole set of such quantities.

The apparatus of fuzzy computing allows us to simply get the membership functions for the characteristics of the averaged stiffness characteristics of the structurally nonhomogeneous masonry material. Thereat we take into account the actual variation of values for the characteristics of the materials of a masonry.

This approach allows us to evaluate the behavior of masonry at various levels of loading and take into account the partial destruction of the masonry components.

In a complex stress-strain state, any strength criterion is formulated in a fuzzy formulation can be used to assess the bearing capacity. The fuzzy number algebra allows us to solve problems that are incomparably more difficult to solve in the probabilistic formulation, and some are generally unsolvable due to insufficient information on the laws of distribution of random variables.

\section{References}

1. SP 15.13330.2017 Stone and Reinforced Masonry Structures (Moscow: Ministry of construction and housing communal service)

2. M. Angelillo, P.B. Lourenço, G. Milani Masonry behaviour and modeling (2014)

3. G.G. Kashevarova, A.J. Zobacheva PNRPU Bulletin. Construction and Architecture 1, 106-116 (2010)

4. O.V. Kabancev, Seismic Resistant Construction. Security of Buildings, 4, 36-40 (2013)

5. V.V. Adishchev, D.S. Shmakov News of Higher Educational Institutions. Construction, 5, 62-73 (2012)

6. V.V. Adishchev, D.S. Shmakov, E.S. Shmakov News of Higher Educational Institutions. Construction, 5, 3-15 (2013)

7. L.A. Zadeh The concept of a linguistic variable and its application to approximate reasoning, University of California, Berkeley (1973)

8. B.S. Reznikov, A.F. Nikitenko, I.V. Kucherenko News of Higher Educational Institutions. Construction, 2, 10-17 (2008)

9. Ceramic brick and stone. General specifications (2012)

10. V.V. Pangaev, V.M. Serdyuk, News of Higher Educational Institutions. Construction, 9, 110-113 (2004)

11. V.V. Adishchev, I.V. Kucherenko, M. S. Gracheva, News of Higher Educational Institutions. Construction, 2-3, 94-102 (2013) 
12. J. M. Emery, J. D. Hochhalther, A. R. Ingraffea, Computational Fracture Mechanics of Concrete Structures: A Retrospective through Multiple Lenses. FraMCos-6 Catania (Italy) (2007)

13. I. V. Kucherenko, V.V. Adishchev, M.S. Teterina, IOP Conf. Series: Materials Science and Engineering, 456 012115, doi:10.1088/1757-899X/456/1/012115 (2018)

14. L.I. Onishchik, Stone structures of industrial and civil buildings (Moscow , 1939) 